\section{ORIGINAL RESEARCH}

A.D. Furtado

B.C. Lau

E. Vittinghoff

W.P. Dillon

W.S. Smith

T. Rigby

L. Boussel

M. Wintermark

\title{
Optimal Brain Perfusion CT Coverage in Patients with Acute Middle Cerebral Artery Stroke
}

BACKGROUND AND PURPOSE: PCT has emerged as an alternative to MR imaging for the assessment of patients with suspected acute stroke. However, 1 disadvantage of PCT is its limited anatomic coverage, which may impact the characterization of hemispheric ischemic strokes. The purpose of this study was to determine the optimal brain CT coverage required to accurately estimate the size of the infarct core relative to the MCA territory and the infarct-penumbra mismatch, by using a criterion standard of these parameters measured on PCT with 80-mm z-axis coverage.

MATERIALS AND METHODS: Fifty-one patients with acute ischemic hemispheric stroke underwent PCT scanning ( 2 boluses, total coverage of $80 \mathrm{~mm}, 16 \times 5 \mathrm{~mm}$ sections) within the first 24 hours of symptom onset and a follow-up NCCT of the brain between 3 days and 3 months after the initial stroke CT study. The volumes of PCT infarct and penumbra for each possible extent of $z$-axis coverage derived from the individual PCT sections were recorded (beginning with $5 \mathrm{~mm}$ of $z$-axis coverage above the orbits and then increasing the coverage in 5-mm increments in the z-axis up to $80 \mathrm{~mm}$ above the orbits). The infarct-penumbra mismatch and the size of the infarction relative to the MCA territory were calculated for each extent of z-axis coverage. Using the $80-\mathrm{mm}$ z-axis coverage as the criterion standard, we calculated the accuracy of the values of the relative PCT infarct size and mismatch that were obtained by using more limited z-axis coverage. The impact of different levels of PCT z-axis coverage on the eligibility for reperfusion treatment was assessed.

RESULTS: On the admission PCT, by using $80-\mathrm{mm}$ of z-axis coverage, the mean perfusion infarct core volume was $45.9 \pm 44.0 \mathrm{~cm}^{3}$ (range, $0-170 \mathrm{~cm}^{3}$ ) and the mean penumbra volume was $64.5 \pm 64.4$ $\mathrm{cm}^{3}$ (range, $0-226 \mathrm{~cm}^{3}$ ). The mean perfusion infarct core/MCA territory ratio was $19.6 \% \pm 16.2 \%$ (range, $0.1 \%-56 \%$ ). The penumbra / (infarct + penumbra) ratio was $68.6 \% \pm 23.6 \%$ (range, $16.4 \%-$ $100 \%$ ). The final infarct volume on follow-up NCCT was $115.4 \pm 157.3 \mathrm{~cm}^{3}$ (range, $1.79-647.4 \mathrm{~cm}^{3}$ ). The minimal z-axis PCT coverage required to obtain values similar to those obtained with $80-\mathrm{mm}$ z-axis coverage was $75 \mathrm{~mm}$ for a mismatch of 0.5 , fifty millimeters for a mismatch of 0.2 , and $55 \mathrm{~mm}$ for a size of PCT infarct relative to the MCA territory.

CONCLUSIONS: Seventy-five millimeters is the minimal PCT coverage required to use PCT as a tool to select patients with acute stroke for reperfusion therapy by using a mismatch of 0.5 . A z-axis coverage of $50 \mathrm{~mm}$ was sufficient for a mismatch of 0.2 ; and $55 \mathrm{~mm}$, for the size of PCT infarct relative to MCA territory (one-third or more).

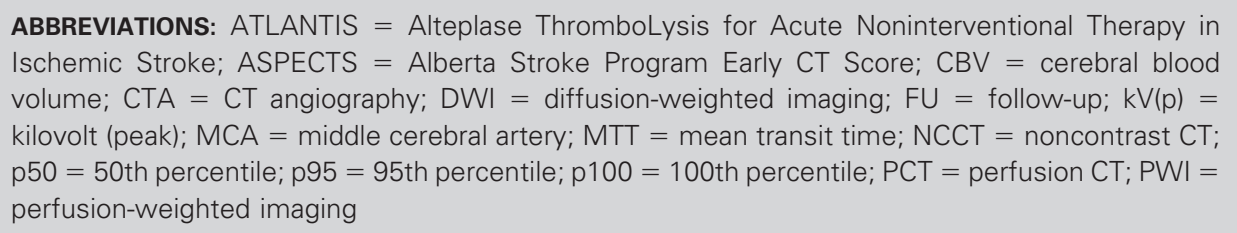

$\mathbf{T}$ he size of the perfusion infarct core relative to the MCA territory and the degree of mismatch (infarct core-penumbra ratio) are typically used to determine eligibility for reperfusion therapy in patients with acute stroke and to select patients for either intravenous or intra-arterial thrombolysis during the extended time window. ${ }^{1-7}$ Although it has been

Received April 27, 2009; accepted after revision July 20.

From the Departments of Radiology (A.D.F., B.C.L., W.P.D., T.R., L.B., M.W.), Neuroradiology Section, Neurology (W.S.S.), and Epidemiology and Biostatistics (E.V.), University of California, San Francisco, California.

Max Wintermark receives funding from the National Center for Research Resources, grant KL2 RR024130, GE Healthcare, and Philips Healthcare.

Please address correspondence to Max Wintermark, MD, University of California, San Francisco, Department of Radiology, Neuroradiology Section, 505 Parnassus Ave, Box 0628, San Francisco, CA 94143-0628; e-mail: Max.Wintermark@radiology.ucsf.edu

Indicates open access to non-subscribers at www.ajnr.org

DOI 10.3174/ajnr.A1880 reported in some clinical trials that patients with stroke and a mismatch ratio of 0.2 (20\%) benefit from acute thrombolysis/ recanalization in an extended time window (3-6 hours), a greater benefit has been shown for a mismatch ratio of 0.5 (50\%). ${ }^{4,8-10}$ The size of the PCT infarct core with low CBV larger than one-third of the MCA territory may represent a contraindication to acute reperfusion therapy because of an increased risk of hemorrhagic transformation. ASPECTS and ATLANTIS have validated the infarct size relative to the MCA territory on admission NCCT of the brain as a predictor of hemorrhagic transformation in patients with ischemic stroke. ${ }^{11}$

PCT has emerged as an alternative to MR imaging, DWI and PWI, for the assessment of patients with suspected acute stroke for reperfusion therapy in an extended time window. ${ }^{12-16}$ The physiologic information obtained from PCT in patients with acute stroke is equivalent to that from DWI/ PWI ${ }^{16-18}$ Finally, PCT and DWI/PWI have also been shown to 
lead to the same treatment decisions regarding the selection of patients with acute ischemic hemispheric strokes for reperfusion therapy. ${ }^{19}$

While PCT studies may miss small ischemic lesions such as lacunar infarcts, PCT has been shown to accurately detect hemispheric ischemic strokes. ${ }^{20,21}$ However, a disadvantage of PCT is its limited anatomic coverage, ${ }^{22,23}$ which may impact the characterization of hemispheric ischemic strokes. With the introduction of 256- and 312-section CT scanners, wholebrain coverage (ie, $8-12 \mathrm{~cm}$ of brain PCT coverage) became possible. Still, the exact benefit of an extended $\mathrm{z}$-axis PCT coverage versus shorter levels of coverage remains unclear.

The purpose of this study was to determine the optimal brain CT coverage required to accurately estimate the size of the infarct core relative to the MCA territory and the infarctpenumbra mismatch, by using a criterion standard of these parameters measured on PCT with $80-\mathrm{mm} \mathrm{z}$-axis coverage.

\section{Materials and Methods}

\section{Study Design}

Imaging data obtained as part of standard clinical stroke care at our institution were retrospectively reviewed with the approval of the institutional review board. At our institution, patients with suspicion of acute stroke and no history of significant renal insufficiency or contrast allergy routinely undergo a stroke CT survey including the following imaging protocol: NCCT, PCT at 2 cross-sectional positions, CTA of the cervical and intracranial vessels, and postcontrast cerebral $\mathrm{CT}$, obtained in this chronologic sequence.

We retrospectively identified a consecutive series of 51 patients admitted to the University of California, San Francisco Medical Center, from January 2006 to June 2007, who met the following inclusion criteria: 1) admission to the emergency department with signs and symptoms suggesting hemispheric stroke within 24 hours after symptom onset, 2) no evidence of intracerebral hemorrhage on the admission NCCT, and 3) evidence of new infarct involving the MCA territory on follow-up NCCT performed within 3 days to 3 months after the admission stroke CT study. Exclusion criteria included evidence of old infarcts on admission NCCT, perfusion deficits in the anterior cerebral artery or posterior cerebral artery distribution, and poor contrast bolus during acquisition of the PCT series. Patients' charts were reviewed for demographic and clinical data.

\section{Imaging Protocol}

PCT studies were performed on a 64-section CT scanner. Each PCT study involved successive gantry rotations performed in cine mode during intravenous administration of iodinated contrast material. Images were acquired and reconstructed at a temporal sampling rate of 1 image per second for the first 45 seconds. Acquisition parameters were $80 \mathrm{kV}(\mathrm{p})$ and $100 \mathrm{mAs}$. Two successive PCT series at 2 different levels were performed following the NCCT and before the CTA. To obtain a $\mathrm{z}$-axis coverage of $80 \mathrm{~mm}, 2$ continuous PCT series were performed without gaps or overlaps, parallel to the hard palate, from the first level above the orbits to avoid the lens. For each PCT level, eight 5-mm-thick CT sections were assessed. The first PCT series was obtained at the level of the third ventricle, the basal ganglia, and the internal capsule; and the second PCT series, $4 \mathrm{~cm}$ above the first one, was obtained at the level of the lateral ventricles, contiguous to the first PCT series. For each PCT series, a 4-mL bolus of iohexol (300$\mathrm{mg} / \mathrm{mL}$ of iodine, Omnipaque; Amersham Health, Princeton, New
Jersey) was administered into an antecubital vein by using a power injector at an injection rate of $5 \mathrm{~mL}$ per second for all patients. CT scanning was initiated 7 seconds after the start of the injection of the contrast bolus.

\section{Image Postprocessing}

PCT data were analyzed using PCT software developed by Extended Brilliance Workstation (Philips Medical Systems Cleveland, Ohio). This software relies on the central volume principle, which is the most accurate for low injection rates of iodinated contrast material. ${ }^{24}$ After motion correction and noise reduction by an anisotropic (ie, edgepreserving spatial) filter, the software applies curve fitting by the least mean squares to obtain mathematic descriptions of the time-attenuation curves for each pixel. A closed-form noniterative deconvolution is then applied to calculate the MTT map. ${ }^{25}$ The deconvolution operation requires a reference arterial input function (most often within the anterior cerebral artery), selected by the PCT software within a region of interest drawn by the user. The CBV map is calculated from the area under the time-attenuation curves. ${ }^{26}$ The PCT infarct core and salvageable brain tissue, as well as their volumes on each PCT section, are automatically calculated by the software by using CBV and MTT thresholds, as reported in the literature (PCT salvageable brain tissue: MTT $>7$ seconds or $145 \%$ of the contralateral side values, $\mathrm{CBV} \geq 2.0 \mathrm{~mL} \times 100 \mathrm{~g}^{-1}$; PCT infarct core: area of $\mathrm{CBV}<2.0$ $\mathrm{mL} \times 100 \mathrm{~g}^{-1} \cdot{ }^{15}$ The volume of the final infarct on FU NCCT was measured manually.

We calculated the contribution of each 5-mm PCT section in the $\mathrm{z}$-axis from $5 \mathrm{~mm}$ above the orbits to $80 \mathrm{~mm}$ of $\mathrm{z}$-axis coverage to estimate infarct-penumbra mismatch and the size of the infarct relative to MCA territory.

The volume of the MCA territory sections was measured from the admission NCCT by using standard anatomic landmarks as described by Barber at al. ${ }^{1}$ and was used to calculate the ratio between perfusion infarct core and MCA territory in percentages. The final infarct volume was calculated on a follow-up NCCT.

\section{Statistical Analysis}

For each patient, we plotted the absolute infarct volume (in milliliters), the PCT infarct core/MCA territory ratio (in percentages), the absolute penumbra volume (in milliliters), and the penumbra / (infarct + penumbra) ratio (in percentages) as a function of the $z$-axis coverage (in millimeters). The value measured for $80-\mathrm{mm} \mathrm{z}$-axis coverage was considered as the criterion standard-maximal coverage obtained.

For each patient and for each of these plots, we calculated if and when a plateau was reached. The measurements for each extent of $\mathrm{z}$-axis coverage were considered successively and compared with the measurements for the $80-\mathrm{mm}$ coverage, and a plateau was defined when the measurements for a shorter $\mathrm{z}$-axis coverage were within $5 \%$ of the corresponding measurement for $80-\mathrm{mm}$ coverage and when the slope with the next coverage $(+5 \mathrm{~mm})$ was $<0.2$. For each parameter (absolute infarct volume in milliliters, the perfusion infarct core/MCA territory ratio in percentages, the absolute penumbra volume in milliliters, and the penumbra / [infarct + penumbra] ratio in percentages), we calculated the coverage for which a plateau was reached for $50 \%$ of the patients (p50), 95\% of the patients (p95), and all (100\%) patients (p100). We reported the p50, p95, and p100 coverage for all patients as a group, as well as for the patients stratified according to absolute penumbra $(<50 \mathrm{~mL}, 50-150 \mathrm{~mL}$, and $>150$ $\mathrm{mL})$, absolute perfusion infarct core $(<25 \mathrm{~mL}, 25-75 \mathrm{~mL}$, and $>75$ 
$\mathrm{mL})$, mismatch $(<20 \%$ and $<50 \%)$, and relative final infarct size $(<33 \%$ and $\geq 33 \%)$.

We then focused on the perfusion infarct core/MCA territory ratio (in percentages) and selected the threshold of 33\%, which is usually used to determine the eligibility of patients with acute stroke for reperfusion therapy. ${ }^{1-7}$ Hypothetic treatment decisions for shorter extents of $\mathrm{z}$-axis coverage were compared with the criterion standard (true-negative if no treatment both at $80-\mathrm{mm}$ coverage and at a shorter coverage; false-negative if treatment at 80 -mm coverage, but no treatment at a shorter coverage; true-positive if treatment both at 80 -mm coverage and at a shorter coverage; false-positive if no treatment at $80-\mathrm{mm}$ coverage, but treatment at a shorter coverage). The sensitivity and specificity of treatment decisions were calculated for each coverage.

Similarly, we calculated the sensitivity and specificity for each extent of $\mathrm{z}$-axis coverage in terms of treatment decisions based on the penumbra / (infarct + penumbra) ratios, with 2 thresholds considered for these ratios (20\% and 50\%): penumbra / (infarct + penumbra) ratios $>20 \%$ or $50 \%$ treatment or positive; penumbra / (infarct + penumbra) ratios $<20 \%$ or $50 \%$ no treatment or negative.

\section{Results}

\section{Patients and Imaging Studies}

A total of 59 patients were studied between January 2006 and June 2007. Eight patients were excluded. Three had poor contrast bolus during the PCT series; 4 had perfusion abnormalities located mainly in the posterior cerebral artery distribution; and one, in the anterior cerebral artery distribution. Of the 51 remaining patients in the analyses, all with evidence of new stroke in the MCA distribution, 29 were men (57\%), with mean age 67.9 years old (range, 31-96). Initial imaging was performed at a median of 7.2 hours after symptom onset, with a range of 0.6-24 hours. Follow-up imaging was performed a median of 4 days after initial imaging, with a range of 3 days to 3 months.

On admission PCT, the mean perfusion infarct core volume was $45.9 \pm 44.0 \mathrm{~cm}^{3}$ (range, $0-170 \mathrm{~cm}^{3}$ ) and the mean penumbra volume was $64.5 \pm 64.4 \mathrm{~cm}^{3}$ (range, $0-226 \mathrm{~cm}^{3}$ ). The mean perfusion infarct core/MCA territory ratio was $19.6 \pm 16.2 \%$ (range, $0.1 \%-56 \%$ ). The penumbra / (infarct + penumbra) was $68.6 \pm 23.6 \%$ (range, $16.4 \%-100 \%$ ). The final infarct volume on FU NCCT was $115.4 \pm 157.3 \mathrm{~cm}^{3}$ (range, $\left.1.79-647.4 \mathrm{~cm}^{3}\right)$.

\section{Influence of PCT Coverage}

The perfusion infarct core/MCA territory volume ratio (Fig 1) and the mismatch penumbras / (penumbra + infarct) ratio (Fig 2) were accurately characterized by using z-axis coverage levels of 75 and $70 \mathrm{~mm}$, respectively. This relatively large extent of required z-axis coverage was driven by patients with large perfusion infarct cores and large penumbras. In patients with small infarct cores or small mismatches, a shorter extent of $\mathrm{z}$-axis coverage was sufficient (Table 1).

The calculations above are for perfusion infarct core/MCA territory ratios and mismatch in general and apply independently of the thresholds used on these parameters. We then considered specific thresholds previously used in clinical trials. ${ }^{2-5}$ A coverage of $55 \mathrm{~mm}$ was sufficient for accuracy in deciding whether the perfusion infarct core was more or less than

\section{Relative Volume of the Baseline Infarct Core Compared to the MCA Territory}

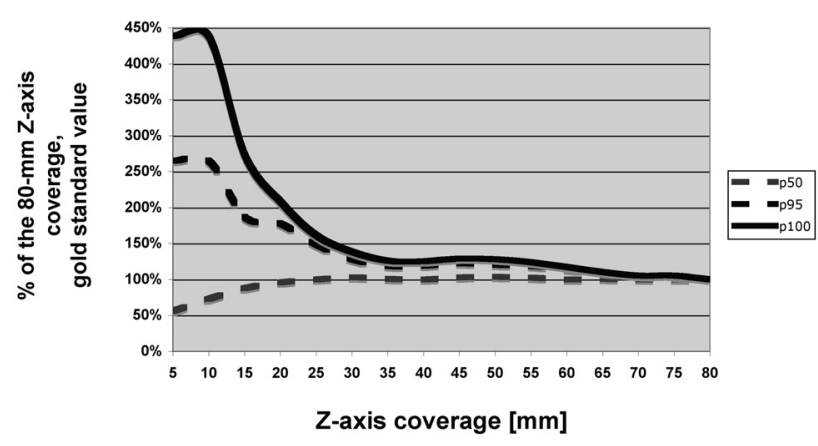

Fig 1. Graph depicts the penumbra-infarct mismatch (penumbra / [penumbra + infarct] ratio). The value measured for $80-\mathrm{mm}$ z-axis coverage was considered as the criterion standard, and the values measured at shorter levels of z-axis coverage are expressed in percentages of the $80-\mathrm{mm}$ z-axis coverage criterion standard value. For short z-axis coverage, the relative baseline infarct volume compared with the volume of the MCA territory is grossly over- or underestimated compared with the relative baseline infarct volume measured at $80-\mathrm{mm}$ z-axis coverage. For $30-\mathrm{mm}$ z-axis coverage, the relative baseline infarct volume is accurately estimated for $50 \%$ (p50) of the patients, but $75-\mathrm{mm}$ $z$-axis coverage is required to accurately assess the relative baseline infarct volume.

\section{Relative Volume of the Baseline Penumbra Compared to the Baseline Infarct Core (Mismatch)}

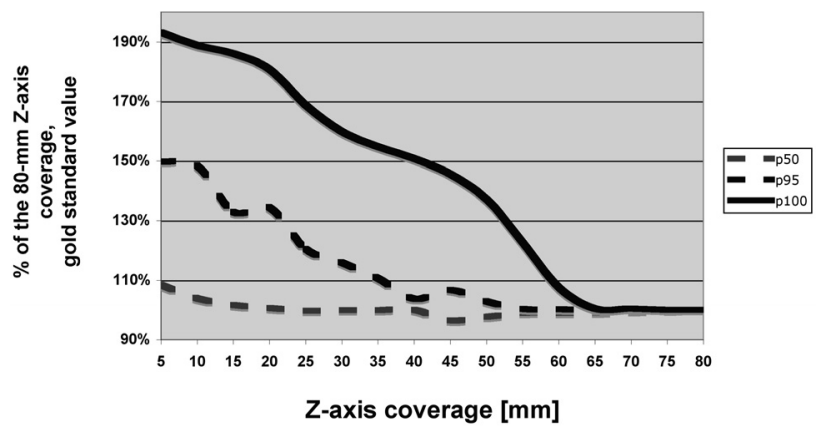

Fig 2. Graph depicts the penumbra-infarct mismatch (penumbra / [penumbra + infarct] ratio). The value measured for $80-\mathrm{mm}$ z-axis coverage was considered as the criterion standard, and the values measured at shorter levels of z-axis coverage are expressed in percentages of the 80-mm z-axis coverage criterion standard value. For shorter levels of $z$-axis coverage, the penumbra-infarct mismatch is grossly overestimated compared with $80-\mathrm{mm}$ z-axis coverage. For $30-\mathrm{mm} z$-axis coverage, the mismatch is accurately estimated for $50 \%$ (p50) of the patients, but $70-\mathrm{mm}$ z-axis coverage is required to assess the penumbra-infarct mismatch accurately.

one-third of the MCA territory (Table 2). A 50-mm coverage was sufficient to accurately characterize a mismatch of $20 \%$ (Table 3 ), while a 75-mm coverage was required to accurately characterize a mismatch of 50\% (Table 4).

\section{Discussion}

Currently, most multidetector scanners offer limited coverage for PCT of the brain. This is often stated as a drawback of PCT compared with whole-brain coverage, for example from MR imaging. ${ }^{22,23}$ Our study quantified the impact of various volumes of z-axis PCT coverage of the brain on the assessment of the volume of the infarct and volume of the infarct-penumbra mismatch, compared with an $80-\mathrm{mm} \mathrm{z}$-axis coverage. PCT $\mathrm{z}$-axis coverage levels of 70 and $75 \mathrm{~mm}$ were equivalent to $80-\mathrm{mm} \mathrm{z}$-axis coverage to estimate mismatch (penumbra / penumbra + infarct) of $50 \%$ and the ratio between the infarct 


\begin{tabular}{|c|c|c|c|c|c|}
\hline Patients & $\begin{array}{l}\text { Number } \\
\text { of Cases }\end{array}$ & $\begin{array}{c}\text { Absolute } \\
\text { Baseline PCT } \\
\text { Infarct Volume }\end{array}$ & $\begin{array}{l}\text { Baseline PCT } \\
\text { Infarct/MCA }\end{array}$ & $\begin{array}{c}\text { Absolute Baseline } \\
\text { PCT Penumbra } \\
\text { Volume }\end{array}$ & $\begin{array}{c}\text { Baseline PCT } \\
\text { Penumbra/ } \\
\text { (Infarct }+ \text { Penumbra) }\end{array}$ \\
\hline All & 51 & 75 & 70 & 75 & 60 \\
\hline \multicolumn{6}{|c|}{ Stratified by baseline absolute PCT infarct volume } \\
\hline$<25 \mathrm{~mL}$ & 19 & 50 & 35 & 65 & 60 \\
\hline $25-75 \mathrm{~mL}$ & 9 & 60 & 40 & 65 & 60 \\
\hline$>75 \mathrm{~mL}$ & 23 & 75 & 70 & 75 & 70 \\
\hline \multicolumn{6}{|c|}{ Stratified by baseline PCT infarct/MCA ratio } \\
\hline$<33 \%$ & 28 & 65 & 40 & 75 & 60 \\
\hline$\geq 33 \%$ & 23 & 75 & 70 & 75 & 70 \\
\hline \multicolumn{6}{|c|}{ Stratified by baseline absolute PCT penumbra volume } \\
\hline$<50 \mathrm{~mL}$ & 14 & 65 & 55 & 70 & 25 \\
\hline $50-150 \mathrm{~mL}$ & 24 & 75 & 55 & 75 & 60 \\
\hline$>150 \mathrm{~mL}$ & 13 & 60 & 65 & 75 & 65 \\
\hline \multicolumn{6}{|c|}{$\begin{array}{l}\text { Stratified by baseline PCT penumbra/ } \\
\text { (infarct }+ \text { penumbra) ratio }\end{array}$} \\
\hline$<20 \%$ & 7 & 15 & 15 & 10 & 10 \\
\hline $20 \%-50 \%$ & 13 & 55 & 55 & 70 & 60 \\
\hline$>50 \%$ & 31 & 75 & 70 & 75 & 70 \\
\hline \multicolumn{6}{|c|}{ Stratified by absolute follow-up infarct volume } \\
\hline$<50 \mathrm{~mL}$ & 22 & 55 & 35 & 65 & 55 \\
\hline $50-100 \mathrm{~mL}$ & 15 & 75 & 50 & 65 & 60 \\
\hline$>100$ & 14 & 70 & 70 & 75 & 70 \\
\hline
\end{tabular}

a Plateau was reached for PCT parameters in 95\% (p95) of patients.

\begin{tabular}{lcc}
\hline $\begin{array}{l}\text { Table 2: Sensitivity and specificity of the brain imaging coverage to } \\
\text { assess PCT infarct size one-third or more of the MCA territory } \\
\text { compared with 80-mm z-axis coverage }\end{array}$ \\
\hline Coverage (mm) & Sensitivity (\%) & Specificity (\%) \\
\hline 5 & 94 & 56 \\
10 & 94 & 69 \\
15 & 97 & 69 \\
20 & 94 & 88 \\
25 & 97 & 88 \\
30 & 97 & 88 \\
35 & 100 & 88 \\
40 & 97 & 88 \\
45 & 100 & 88 \\
50 & 100 & 88 \\
$\mathbf{5 5}$ & $\mathbf{1 0 0}$ & $\mathbf{1 0 0}$ \\
$\mathbf{6 0}$ & $\mathbf{1 0 0}$ & $\mathbf{1 0 0}$ \\
$\mathbf{6 5}$ & $\mathbf{1 0 0}$ & $\mathbf{1 0 0}$ \\
$\mathbf{7 0}$ & $\mathbf{1 0 0}$ \\
$\mathbf{7 5}$ & $\mathbf{1 0 0}$ & $\mathbf{1 0 0}$ \\
80 (criterion standard) & $\mathbf{1 0 0}$ & $\mathbf{1 0 0}$ \\
\hline
\end{tabular}

Note:- Bold numbers indicate the PCT coverages for which a 100\% sensitivity and a 100\% specificity are achieved.

on PCT and the MCA territory, respectively. A z-axis coverage of $50 \mathrm{~mm}$ was adequate to characterize whether the mismatch was $\leq 20 \%$; and a $\mathrm{z}$-axis coverage of $55 \mathrm{~mm}$, to determine whether the infarct on perfusion was one-third of the MCA territory or more.

This finding has practical implications in terms of PCT imaging acquisition because 2 boluses of contrast injection with 2 separate acquisitions by using a 64-section scanner are needed for 75-mm coverage. Alternatively, other techniques of CT acquisition such as volume shuttles could be used to achieve $\mathrm{z}$-axis coverage with a single contrast bolus injection. Large $\mathrm{z}$-axis coverage with a single bolus can also be obtained with a single bolus by using a 256- or a 320 -section CT scanner.

\begin{tabular}{lcc}
\hline $\begin{array}{l}\text { Table 3: Sensitivity and specificity of the brain imaging coverage to } \\
\text { assess PCT penumbra / (infarct } \\
\text { with } \mathbf{8 0} \text { - } \mathbf{m m} \text { z-axis coverage }\end{array}$ & \\
\hline Coverage (mm) & Sensitivity (\%) & Specificity $(\%)$ \\
\hline 5 & 95 & 91 \\
10 & 95 & 91 \\
15 & 95 & 91 \\
20 & 95 & 91 \\
25 & 95 & 100 \\
30 & 98 & 91 \\
35 & 95 & 100 \\
40 & 98 & 100 \\
45 & 98 & 100 \\
$\mathbf{5 0}$ & $\mathbf{1 0 0}$ & $\mathbf{1 0 0}$ \\
$\mathbf{5 5}$ & $\mathbf{1 0 0}$ & $\mathbf{1 0 0}$ \\
$\mathbf{6 0}$ & $\mathbf{1 0 0}$ & $\mathbf{1 0 0}$ \\
$\mathbf{6 5}$ & $\mathbf{1 0 0}$ & $\mathbf{1 0 0}$ \\
$\mathbf{7 0}$ & $\mathbf{1 0 0}$ & $\mathbf{1 0 0}$ \\
$\mathbf{7 5}$ & $\mathbf{1 0 0}$ & $\mathbf{1 0 0}$ \\
$\mathbf{8 0}$ (criterion standard) & $\mathbf{1 0 0}$ & $\mathbf{1 0 0}$ \\
\hline
\end{tabular}

Note:-Bold numbers indicate the PCT coverages for which a 100\% sensitivity and a $100 \%$ specificity are achieved.

The marked variation between the p95 and p100 curves in Fig 2 suggests the presence of a group of outliers. This finding seems to be secondary to a reasonable number of patients with distal MCA branch infarctions, which are frequently located more cranially; and a more extended coverage was needed either for qualitative or quantitative analysis. Because the presentation of these 2 groups is indistinguishable clinically, a study involving only the patients with more proximal MCA occlusions would have little clinical relevance.

Studies using a 256- or a 320-section CT scanner to show the benefits of higher levels of $\mathrm{z}$-axis coverage are yet to be concluded as are large clinical trails to determine the best level of the mismatch and the value perfusion infarct size relative to the MCA territory in acute ischemic stroke. However, accord- 


\begin{tabular}{|c|c|c|}
\hline Coverage (mm) & Sensitivity (\%) & Specificity (\%) \\
\hline 5 & 87 & 85 \\
\hline 10 & 87 & 85 \\
\hline 15 & 87 & 85 \\
\hline 20 & 87 & 90 \\
\hline 25 & 90 & 85 \\
\hline 30 & 94 & 90 \\
\hline 35 & 94 & 90 \\
\hline 40 & 90 & 95 \\
\hline 45 & 94 & 90 \\
\hline 50 & 94 & 95 \\
\hline 55 & 94 & 95 \\
\hline 60 & 97 & 100 \\
\hline 65 & 94 & 95 \\
\hline 70 & 97 & 100 \\
\hline 75 & 100 & 100 \\
\hline 80 (criterion standard) & 100 & 100 \\
\hline
\end{tabular}

ing to our study, it seems that more extended levels of z-axis coverage have little if any contribution to estimating a mismatch of 0.5 , a mismatch of 0.2 , and the size of PCT infarct relative to the MCA territory (equal or more than one-third).

We acknowledge some limitations to our study. Notably, our study focused on a relatively small sample of patients. However, it encompassed a wide range in volumes of baseline infarct, baseline mismatch, and final infarct volume. The median time from symptom onset was 7.2 hours, which is beyond the time window for intravenous reperfusion therapy. This may limit the generalizability of our results.

Our criterion standard was suboptimal. It is unclear how accurately $80-\mathrm{mm} \mathrm{z}$-axis coverage compares with whole-brain coverage. However, if $75 \mathrm{~mm}$ is the minimal PCT coverage required to compare accurately with an 80 -mm criterion standard, it is reasonable to assume that a coverage of $\geq 75 \mathrm{~mm}$ would be needed to compare with whole-brain coverage.

There are theoretically an extremely high number of section combinations that could have been tested for each coverage. Testing all these combinations was beyond our goal. Rather, we decided to focus on reproducing the different approaches typically used in institutions and reported in the literature, namely 1 or 2 sections of PCT above the orbits (with 1 bolus on a 16-section scanner), 8 sections above the orbits (with 1 bolus on a 64-section scanner), and 2 boluses or the toggling-table technique.

Finally, only 1 PCT software package was used for this study, and the results may not hold true for other software packages.

\section{Conclusions}

$\mathrm{Z}$-axis coverage of $75 \mathrm{~mm}$ is the minimal PCT coverage required to use PCT as a tool to select patients with acute stroke for reperfusion therapy by using a mismatch of 0.5 . A z-axis coverage of $50 \mathrm{~mm}$ was sufficient for a mismatch of 0.2 ; and 55 $\mathrm{mm}$, for a relative size of PCT infarct to MCA territory (onethird or more).

\section{References}

1. Barber PA, Demchuk AM, Zhang J, et al. Validity and reliability of a quantitative computed tomography score in predicting outcome of hyperacute stroke before thrombolytic therapy: ASPECTS Study Group Alberta Stroke Programme Early CT Score. Lancet 2000;355:1670-74

2. Furlan AJ, Eyding D, Albers GW, et al. Dose Escalation of Desmoteplase for Acute Ischemic Stroke (DEDAS): evidence of safety and efficacy 3 to 9 hours after stroke onset. Stroke 2006;37:1227-31. Epub 2006 Mar 1230

3. Hacke W, Albers G, Al-Rawi Y, et al. The Desmoteplase in Acute Ischemic Stroke Trial (DIAS): a phase II MRI-based 9-hour window acute stroke thrombolysis trial with intravenous desmoteplase. Stroke 2005;36:66-73. Epub 2004 Nov 2029

4. Albers GW, Thijs VN, Wechsler L, et al. Magnetic resonance imaging profiles predict clinical response to early reperfusion: the diffusion and perfusion imaging evaluation for understanding stroke evolution (DEFUSE) study. Ann Neurol 2006;60:508-17

5. Davis SM, Donnan GA, Parsons MW, et al. Effects of alteplase beyond $\mathbf{3} \mathbf{~ h}$ after stroke in the Echoplanar Imaging Thrombolytic Evaluation Trial (EPITHET) a placebo-controlled randomised trial. Lancet Neurol 2008;7:299-309. Epub 2008 Feb 28

6. Muir KW, Baird-Gunning J, Walker L, et al. Can the ischemic penumbra be identified on noncontrast CT of acute stroke? Stroke 2007;38:2485-90. Epub 2007 Aug 2

7. Parsons MW, Pepper EM, Bateman GA, et al. Identification of the penumbra and infarct core on hyperacute noncontrast and perfusion CT. Neurology 2007;68:730-36

8. Luby M, Warach S. Reliability of MR perfusion-weighted and diffusionweighted imaging mismatch measurement methods. AJNR Am J Neuroradiol 2007;28:1674-78. Epub 2007 Sep 20

9. Butcher KS, Parsons M, MacGregor L, et al. Refining the perfusion-diffusion mismatch hypothesis. Stroke 2005;36:1153-59

10. Kakuda W, Lansberg MG, Thijs VN, et al. Optimal definition for PWI/DWI mismatch in acute ischemic stroke patients. J Cereb Blood Flow Metab 2008;28: 887-91. Epub 2008 Jan 9

11. Larrue V, von Kummer R, del Zoppo G, et al. Hemorrhagic transformation in acute ischemic stroke: potential contributing factors in the European Cooperative Acute Stroke Study. Stroke 1997;28:957-60

12. Wintermark $M$, Bogousslavsky J. Imaging of acute ischemic brain injury: the return of computed tomography. Curr Opin Neurol 2003;16:59-63

13. Barber PA, Hill MD, Eliasziw M, et al. Imaging of the brain in acute ischaemic stroke: comparison of computed tomography and magnetic resonance diffusion-weighted imaging. J Neurol Neurosurg Psychiatry 2005;76:1528-33

14. Rai AT, Carpenter JS, Peykanu JA, et al. The role of CT perfusion imaging in acute stroke diagnosis: a large single-center experience. J Emerg Med 2008;4:4

15. Wintermark M, Flanders AE, Velthuis B, et al. Perfusion-CT assessment of infarct core and penumbra: receiver operating characteristic curve analysis in 130 patients suspected of acute hemispheric stroke. Stroke 2006;37:979-85. Epub 2006 Mar 2

16. Schramm P, Schellinger PD, Klotz E, et al. Comparison of perfusion computed tomography and computed tomography angiography source images with perfusion-weighted imaging and diffusion-weighted imaging in patients with acute stroke of less than $\mathbf{6}$ hours' duration. Stroke 2004;35:1652-58. Epub 2004 May 20

17. Wintermark M, Reichhart M, Cuisenaire O, et al. Comparison of admission perfusion computed tomography and qualitative diffusion- and perfusionweighted magnetic resonance imaging in acute stroke patients. Stroke 2002;33:2025-31

18. Na DG, Ryoo JW, Lee KH, et al. Multiphasic perfusion computed tomography in hyperacute ischemic stroke: comparison with diffusion and perfusion magnetic resonance imaging. J Comput Assist Tomogr 2003;27:194-206

19. Wintermark M, Meuli R, Browaeys P, et al. Comparison of CT perfusion and angiography and MRI in selecting stroke patients for acute treatment. Neurology 2007;68:694-97

20. Wintermark M, Fischbein NJ, Smith WS, et al. Accuracy of dynamic perfusion CT with deconvolution in detecting acute hemispheric stroke. AJNR Am J Neuroradiol 2005;26:104-12

21. Esteban JM, Cervera V. Perfusion CT and angio CT in the assessment of acute stroke. Neuroradiology 2004;46:705-15

22. Lin K, Rapalino O, Law M, et al. Accuracy of the Alberta Stroke Program Early CT Score during the first 3 hours of middle cerebral artery stroke: comparison of noncontrast CT, CT angiography source images, and CT perfusion. AJNR Am J Neuroradiol 2008;29:931-36. Epub 2008 Feb 13

23. Miles KA, Griffiths MR. Perfusion CT: a worthwhile enhancement? Br J Radiol 2003;76:220-31

24. Wintermark M, Maeder P, Thiran JP, et al. Quantitative assessment of regiona cerebral blood flows by perfusion CT studies at low injection rates: a critical review of the underlying theoretical models. Eur Radiol 2001;11:1220-30

25. Axel L. Tissue mean transit time from dynamic computed tomography by a simple deconvolution technique. Invest Radiol 1983;18:94-99

26. Zilkha E, Ladurner G, Iliff LD, et al. Computer subtraction in regional cerebral blood-volume measurements using the EMI-scanner. Br J Radiol 1976;49: $330-34$ 